\title{
A special issue on nanotoxicology
}

\author{
C. Weiss $\cdot$ S. Diabaté
}

(C) Springer-Verlag 2011

It is a great pleasure to introduce this special issue on nanotoxicology. This novel discipline of toxicology addresses potential adverse effects of manufactured nanomaterials in biological systems. As the number of manufactured nanomaterials is ever growing, a huge variety of materials needs to be tested for safety now and in the future. Therefore, toxicological research in this area is of utmost importance and reemphasizes the relevance of toxicology in general. The attractiveness of nanotoxicology for researchers lies in all the basic research questions immanent to a young and emerging area in science. As is in general true for toxicology, nanotoxicology in particular is a highly interdisciplinary field, which brings together scientists from totally different backgrounds such as physics, chemistry, biology, engineering, medicine and toxicology, to name a few. Nanotoxicology is not only relevant to assess the risk of unintended nanomaterial exposure but will also help to support the development of nanomedicine by prevention of side effects of pharmaceutical nanoproducts such as those intended for drug delivery, diagnostics or cancer treatment.

The goal of this special issue was to compile the most recent views and work of leading experts in nanotoxicology by a series of timely reviews followed by original research articles. The first review summarizes our knowledge on the toxicity of quantum dots (QDs) with a special interest in the role of surface modifications (Hoshino et al. 2011, this issue). As QDs are superior for labelling

C. Weiss $(\bowtie) \cdot$ S. Diabaté

Karlsruhe Institute of Technology (KIT),

Campus North Institute of Toxicology and Genetics,

Hermann-von-Helmholtz-Platz 1,

76344 Eggenstein-Leopoldshafen, Germany

e-mail: carsten.weiss@kit.edu biomolecules as well as cells, such as stem and cancer cells, they are promising tools for basic research and nanomedicine, provided that their inherent toxicity is prevented. The advantage of QDs is their traceability and the possibility to quantify their dose, an issue which is a major problem for most other, non-labelled nanomaterials. The next review addresses this very important aspect of labelling nanomaterials with radioisotopes, which hopefully will allow for the proper quantification of nanomaterials in different biological environments and pave the way for the proper dosimetry of nanomaterials (Gibson et al. 2011, this issue). Similarly, the choice of model systems for risk assessment is an ongoing debate. Naturally, in vitro systems are currently explored due to their simplicity, higher throughput and as an alternative to animal experiments. However, whether in vitro models are really predictive for the in vivo situation is discussed in the review by Clift and colleagues (Clift et al. 2010, this issue). The series of reviews is finished by an overview of an even less understood topic in nanotoxicology namely mechanisms (Marano et al. 2010, this issue). The so far known cellular targets and signal transduction pathways affected by nanomaterials are reviewed, and this is certainly just the beginning of a fascinating journey to unravel mechanisms of actions in nanotoxicology.

Finally, a series of original research articles conclude this issue. Due to their industrial and potential biomedical relevance, mostly inorganic nanomaterials are studied with the exception of one paper on the toxicity of carbon nanotubes. The first contribution investigates the sizedependent localization and uptake of labelled silica particles (Al-Rawi et al. 2011, this issue) followed by three studies on the cyto- and genotoxicity of silver nanoparticles (Foldbjerg et al. 2010, this issue), platinum nanoparticles (Gehrke et al. 2011, this issue) and carbon 
nanotubes (Kim et al. 2010, this issue). The last two papers investigate the response to nanomaterials in vivo. Whereas in the first study inflammation and irritation is monitored in the mouse after inhalative exposure to titanium dioxide nanoparticles (Leppänen et al. 2011, this issue), the second article makes use of the ecotoxicological model organism zebrafish to explore the response to lead sulphide nanoparticles coated with different ligands (Truong et al. 2010, this issue).

At the end, we would like to thank the editorial team, especially Beate Graf and Jan Hengstler, not only for the invitation to compose such a special issue but also for their constant support throughout the process. Last but not least, we express our gratitude to all authors for their fine contributions and hope that all readers will also enjoy this special issue on nanotoxicology.

\section{References}

Al-Rawi M, Diabaté S, Weiss C (2011) Uptake and intracellular localization of submicron and nano-sized $\mathrm{SiO}_{2}$ particles in $\mathrm{HeLa}$ cells. Arch Toxicol. doi:10.1007/s00204-010-0642-5 (this issue)

Clift MJD, Gehr P, Rothen-Rutishauser B (2010) Nanotoxicology: a perspective and discussion of whether or not in vitro testing is a valid alternative. Arch Toxicol. doi:10.1007/s00204-010-0560-6 (this issue)
Foldbjerg R, Dang DA, Autrup H (2010) Cytotoxicity and genotoxicity of silver nanoparticles in the human lung cancer cell line, A549. Arch Toxicol. doi:10.1007/s00204-010-0545-5 (this issue)

Gehrke H, Pelka J, Hartinger CG, Blank H, Bleimund F, Schneider R, Gerthsen D, Bräse S, Crone M, Türk M, Marko D (2011) Platinum nanoparticles and their cellular uptake and DNA platination at non-cytotoxic concentrations. Arch Toxicol. doi: 10.1007/s00204-010-0636-3 (this issue)

Gibson N, Holzwarth U, Abbas K, Simonelli F, Kozempel, Cydzik I, Cotogno G, Bulgheroni A, Gilliland D, Ponti J, Franchini F, Marmorato P, Stamm H, Kreyling W, Wenk A, Semmler-Behnke M, Buono S, Maciocco L, Burgio N (2011) Radiolabelling of engineered nanoparticles for in vitro and in vivo tracing applications using cyclotron accelerators. Arch Toxicol (this issue)

Hoshino AF, Hanada S, Yamamoto K (2011) Toxicity of nanocrystal quantum dots: the relevance of surface modifications. Arch Toxicol (this issue)

Kim JS, Lee K, Lee YH, Cho HS, Kim KH, Choi KH, Lee SH, Song KS, Kang CS, Yu IJ (2010) Aspect ratio has no effect on genotoxicity of multi-wall carbon nanotubes. Arch Toxicol. doi: 10.1007/s00204-010-0574-0 (this issue)

Leppänen M' Korpi A, Miettinen M, Leskinen J, Torvela T, Rossi EM, Vanhala E, Wolff H, Alenius H, Kosma VM, Joutsensaari J, Jokiniemi J, Pasanen P (2011) Nanosized $\mathrm{TiO}_{2}$ caused minor airflow limitation in the murine airways. Arch Toxicol. doi: 10.1007/s00204-011-0644-y (this issue)

Marano F, Hussain S, Rodrigues-Lima F, Baeza-Squiban A, Boland S (2010) Nanoparticles: molecular targets and cell signalling. Arch Toxicol. doi: 10.1007/s00204-010-0546-4 (this issue)

Truong L, Moody IS, Stankus DP, Nason JA, Lonergan MC, Tanguay RL (2010) Differential stability of lead sulfide nanoparticles influences biological responses in embryonic zebrafish. Arch Toxicol. doi:10.1007/s00204-010-0627-4 (this issue) 